\title{
Ketogenic diet and testosterone increase: Is the increased cholesterol intake responsible? To what extent and under what circumstances can there be benefits?
}

\author{
Heitor Oliveira Santos
}

School of Medicine, Federal University of Uberlandia (UFU), Uberlandia, Minas Gerais, Brazil

\section{DIETARY CHOLESTEROL FROM \\ THE KETOGENIC DIET: IS IT A NEW SOURCE OF INCREASED TESTOSTERONE?}

The new study by Jacob Wilson et al demonstrated that a nutritional intervention based on the ketogenic diet (KD) in resistance-trained athletes of college age showed an increase of total testosterone compared to individuals who followed a Western diet (WD) protocol with the same resistance training. The total testosterone increase in the KD group was $\sim 570 \mathrm{ng} / \mathrm{dL}$ to $\sim 690 \mathrm{ng} / \mathrm{dL}$ after 12 weeks of intervention, while there was no change in free testosterone. ${ }^{1}$ The question is whether this concerned an impactful biological increase, while also of importance is determination of what factor elevated total testosterone in these individuals.

In Wilson's study, both the KD and the WD groups followed an isocaloric diet, meaning that the differential was related to the increase of total lipids and dietary cholesterol and the decrease in dietary fiber: as expected, total carbohydrates in the $\mathrm{KD}$ group also decreased. ${ }^{1}$ However, with regard to these nutrients dietary cholesterol was not calculated, despite the

Address for correspondence:

Heitor Oliveira Santos School of Medicine, Federal University of Uberlandia (UFU), Uberlandia, Minas Gerais, Brazil; Avenue Pará 1720, district Umuarama, Uberlândia - MG; Tel.: (55) 34 3225-8604, Fax: (55) 34 3232-8620, E-mail: heitoroliveirasantos@gmail.com

Received: 16-03-2017, Accepted: 30-03-2017 fact that cholesterol is known to be physiologically a matrix in the formation of androgens, and especially testosterone. $^{2}$

The dietary cholesterol in Wilson's KD subjects was greater than that of the WD group. ${ }^{1}$ In parallel, given that the total lipid intake in the KD was approximately $220 \mathrm{~g}$ per day, and within this total $110 \mathrm{~g}$ was of saturated fat, ${ }^{1}$ dietary cholesterol was also higher than traditional daily recommendations $(<300 \mathrm{mg} / \mathrm{d}$ for healthy individuals $) .^{3}$

Furthermore, while it is normal to have increased intake of dietary cholesterol through the ingestion of the food rich in fat used in the KD, Wilson et al did not quantify dietary cholesterol in their study. Meanwhile, another recent study ${ }^{4}$ quantified daily dietary cholesterol intake in a KD protocol in which there was a similar nutritional ratio to that of Wilson et al and the value obtained was $\sim 495 \mathrm{mg}$, thus providing better insight. ${ }^{1,4}$ Moreover, in the study by Volek et al, the cholesterol intake with a KD was $844 \pm 351 \mathrm{mg} / \mathrm{d}$, thus there were those whose intake of cholesterol was more than $1000 \mathrm{mg} / \mathrm{d} .^{5}$

\section{DOES THE DECREASE IN FIBER INTAKE POTENTIATE THE INCREASE OF TESTOSTERONE THROUGH THE KETOGENIC DIET?}

Besides dietary cholesterol, of considerable importance in this context is dietary fiber. It is known 
that dietary fibers, especially the soluble type, act in the enterohepatic cycle thereby increasing the fecal excretion of cholesterol, a factor that must be considered. ${ }^{6}$

With this in mind, in the study by Wilson et al the KD group ingested fewer dietary fibers than did the WD group ( $18 \mathrm{~g}$ vs $35 \mathrm{~g} / \mathrm{d}) .{ }^{1}$ On the other hand, a large classical study reported that after adjustments of factors with potential for confusion (e.g., age, sex, body weight, body mass index and alcohol intake), there was no difference in total testosterone in individuals with high fiber intake compared to individuals who followed a typical omnivorous diet. ${ }^{7}$ The individuals whose fiber intake was high were vegans. In this group, daily dietary questionnaires over a period of 4 days determined that an average of $57 \mathrm{~g} / \mathrm{d}$ of dietary fiber was achieved, whereas the daily dietary fiber intake among omnivorous individuals averaged $35 \mathrm{~g}^{7}$, this value being equal to that of the WD group of Wilson's study. ${ }^{1}$

Thus, based on the classic study ${ }^{7}$ it may be ascertained that the fact that the dietary fiber intake in Wilson's KD group was lower than that of the group following a traditional diet was not the factor differential which brought about the increase in total testosterone. ${ }^{1}$ Although in the classic study no difference was detected in testosterone levels between the vegan group and the WD group, the KD may involve a higher cholesterol intake than the WD. It is therefore possible that the cholesterol intake of those following a WD is not enough to increase testosterone and, in fact, it would appear necessary to ingest an amount of cholesterol compatible to that of the KD to increase testosterone. $^{1}$

\section{BODY COMPOSITION: DOES INCREASE OF TESTOSTERONE THROUGH THE KETOGENIC DIET ACCOUNT FOR BODY COMPOSITION CHANGES?}

Although there is evidence that testosterone reduces body fat loss, ${ }^{8}$ there is no confirmation to date that the increase of total testosterone through a KD is a powerful factor in attaining this goal. This is because the main factor contributing to body fat loss through a $\mathrm{KD}$ is the caloric deficit and, in parallel, the decrease of insulinemia and mitochondrial modulation. ${ }^{9}$
Several studies have shown that following a KD results in loss of body weight, including fat mass, as well as reduction in body water. ${ }^{9-13}$ However, the latter diet programs are characterized by a high caloric deficit, for example, $\sim 1200 \mathrm{kcal} / \mathrm{day},{ }^{9}$ hence, it cannot to be affirmed that the loss of body fat occurs because of the increase in endogenous testosterone.

Wilson et al demonstrated that the isocaloric KD did not decrease lean mass, strength and potency of upper and lower limbs as compared with the WD. Meanwhile, the increase of testosterone in the KD group was probably not the chief factor in maintaining muscle mass. Instead, it was the strict supervision exerted of the subjects' physical exercise program and the quality and quantity of a diet in which calories and proteins were not restricted. ${ }^{1}$

The increase of testosterone achieved through the KD does not constitute a method of an anabolic-like but doping-free muscular effect, since the traditional protocol to achieve ketosis is limited to protein intake. In fact, the protein intake in the KD is counterproductive in achieving a perfect anabolic metabolism, given that current recommendations for athletes encourage almost double the proportion of protein intake compared to that of the ketogenic diet. ${ }^{14,15}$

\section{CAN THE KETOGENIC DIET LEAD TO ATHEROSCLEROSIS? DOES IT IMPACT DYSLIPIDEMIA?}

There is a paucity of reports in the literature regarding the atherogenic effects induced by the KD. First, there is no long-term intervention, covering for example several years, and no study thus far has analyzed atheroma plaques in the context of a KD. Secondly, most of the KD intervention studies show a favorable or neutral effect on modulation of lipid profile. ${ }^{9,11,16}$

A hypocaloric KD in overweight individuals may decrease LDL, total cholesterol and triglycerides while increasing HDL ( $+5 \mathrm{mg} / \mathrm{dL}) .{ }^{10,11}$ A study published in the Annals of Internal Medicine showed a remarkable decrease of $\sim 105 \mathrm{mg} / \mathrm{dL}$ of serum triglycerides in obese subjects undergoing 6 months of the KD, 
an effect equivalent to that of fibrates. ${ }^{11}$ However, in the study by Urbain et al there was an increase in LDL ( $+12 \mathrm{mg} / \mathrm{dL})$ and total cholesterol $(+9 \mathrm{mg} / \mathrm{dL})$ in healthy subjects undergoing a 6-week normocaloric $\mathrm{KD}$, though this did not exceed the reference range. Moreover, HDL and serum triglycerides did not change. ${ }^{4}$

It is thus clear that with regard to the traditional lipid profile (i.e., total cholesterol, HDL, LDL and triglycerides), the main problem with the KD would appear to be its effect on serum cholesterol. ${ }^{4}$ A hypocaloric KD is associated with an improvement of lipid profile, this most probably attributable to loss of body fat and carbohydrate restriction. However, when the KD is normocaloric it does not appear to alter the lipid profile to any significant degree, since lipid levels did not exceed the reference range. ${ }^{9,11}$

The increase in cholesterol intake exerts a minimal effect on serum cholesterol; ${ }^{17,18}$ therefore, the KD should not be considered a factor significantly affecting the lipid profile. However, it is conceivable that the $\mathrm{KD}$, which is by definition hypercaloric, may negatively affect the lipid profile: interventions in this field are necessary in order to verify this fact. In these cases it is important to assess the levels of LDL for its atherogenic potential, i.e. to examine the types of emerging LDL molecules regarding their size and density following a KD diet.

Furthermore, given that atherosclerosis is a process induced by an antioxidant imbalance, the KD can be of the antioxidant type. For example, there is a type of Mediterranean KD which is mainly composed of omega-9 chiefly due to the consumption of olive oil, omega-3 derived from fish, various sources of vegetables and eggs: ${ }^{9,10}$ there is a consensus that all these foods have antioxidant and anti-inflammatory potentials. ${ }^{3,19-21}$ Therefore, if individuals follow a KD with good dietary sources such as those mentioned above, it may even confer cardiovascular protection even with high cholesterol intake. It is important to stress that the KD modulates the antioxidant genes and mitochondrial metabolism through ketone bodies as signaling metabolites..$^{22}$

\section{STATINS CAN NEGATE THE POSSIBLE INCREASE OF TESTOSTERONE THROUGH THE KETOGENIC DIET (Figure 1)}

Statins (HMG-CoA reductase inhibitors) could potentially decrease testosterone levels by reducing the availability of cholesterol for androgen synthesis. There is a high prevalence of hypogonadism in men with type 2 diabetes and in such patients KD planning may be beneficial for the improvement of glucose metabolism. By contrast, type 2 diabetes is a disease closely related to dyslipidemia, the treatment of which often leads to the use of statins..$^{23,24}$ Therefore, the use of statins in a KD program could be counterproductive since reducing cholesterol can increase testosterone.

In a study carried out by Stanworth et al, type 2 diabetic subjects $(n=147)$ undergoing statin therapy

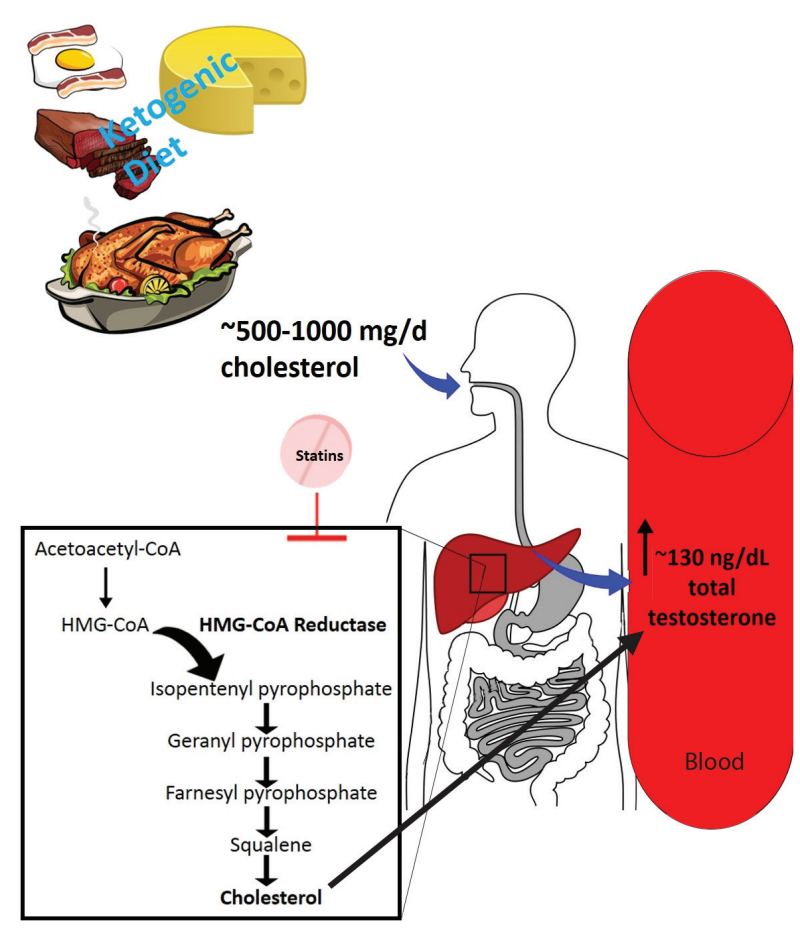

Figure 1. In general, it is expected to consume between 500$100 \mathrm{mg} / \mathrm{d}$ of cholesterol through the ketogenic diet, and may vary beyond this range. Probably after gastrointestinal tract way this cholesterol amount provides more substrate for androgen formation, thus reflecting on a serum increase of total testosterone. Statins can this increase in testosterone by inhibiting the cholesterol pathway. HMG-CoA: 3-Hydroxy-3-methylglutaryl-coenzyme A. 
were seen to have lower total testosterone compared with non-statin users $(\mathrm{n}=167)$ ( $343 \mathrm{vs} .386 \mathrm{ng} / \mathrm{dL}$ of total testosterone, $\mathrm{p}=0.006) .{ }^{24} \mathrm{~A}$ sub-analysis showed that atorvastatin was associated with reduced total testosterone compared with non-statin users ( 329 vs. $386 \mathrm{ng} / \mathrm{dL} ; \mathrm{p}=0.006$ ), while there was an apparent dose-response effect with the lowest levels of total testosterone reported in men treated with $20 \mathrm{mg}$ of atorvastatin compared with the non-statin users (277 $\mathrm{ng} / \mathrm{dL}$ vs. $386 \mathrm{ng} / \mathrm{dL} ; \mathrm{p}=0.017)$. Therefore, based on the use of $20 \mathrm{mg}$ of atorvastatin as an example, there is a decrease of around $109 \mathrm{ng} / \mathrm{dL}$ of total testosterone through use of this drug, this indicating that it may negate the potential increase of total testosterone in the $\mathrm{KD}$ program. ${ }^{24}$

Among 4,166 men, those who used statins were reported to have significantly lower total testosterone levels as compared to non-users, with adjustment according to age, body mass index, cardiovascular disease, diabetes mellitus, hypertension and estradiol levels. The use of several types of statins (simvastatin, pravastatin, fluvastatin, atorvastatin and rosuvastatin) for a period of 1 to 6 months was sufficient to lower total testosterone levels. ${ }^{25}$ In a meta-analysis studying 5 homogeneous trials of $501 \mathrm{men}$, statins lowered testosterone by $19 \mathrm{ng} / \mathrm{dL}$ ( $95 \%$ confidence interval, -0.14 to -1.18 ) mainly in middle-aged subjects with hypercholesterolemia. ${ }^{23}$

\section{THE KETOGENIC DIET IN TYPE 2 DIABETES AND METABOLIC SYNDROME}

Of interest, along with considering a $\mathrm{KD}$ as a support in increasing levels of testosterone, the same diet could be valuable in the treatment of metabolic syndrome and type 2 diabetes mellitus. ${ }^{26}$ For optimal planning of a KD, Paoli et al recommended a fasting blood glucose range of $65-80 \mathrm{mg} / \mathrm{dL}$ and $80-120 \mathrm{mg} / \mathrm{dL}$ for a Western type of diet; in relation to insulinemia, they cite a range between 6-9.4 $\mathrm{mU} / \mathrm{L}$ for the $\mathrm{KD}$ and $6-23 \mathrm{mU} / \mathrm{L}$ in a typically WD. ${ }^{16}$

In the context of this new development, the potential of the KD to increase testosterone may be a therapeutic adjunct for the treatment of other conditions, as for instance for patients with type 2 diabetics who exhibit less testosterone than healthy individuals. ${ }^{27}$
Thus, the KD may be a complement to the therapy of this disease for a certain period of time and may provide some increase of testosterone in parallel to the glycemic control needed in these individuals.

\section{WHAT IS MISSING?}

All things considered, Wilson's study indirectly provides a solid basis for further research into planning a dietary method for boosting testosterone production in men with less than appropriate testosterone levels. ${ }^{1}$

To ascertain whether the possible increase in testosterone through the $\mathrm{KD}$ is derived from the general eating plan of this diet (i.e. high intake of total and saturated fats and cholesterol, and low in carbohydrates), or if it stems uniquely from the increased cholesterol intake, a controlled intervention is necessary based on adjusting for each dietary factor, especially the amount of total fat.

In addition, further studies should determine specifically which KD foods provide dietary cholesterol, for example, whether a majority portion comes from egg yolk or from particular types of animal fats, since certain foods, though high in cholesterol, may supply excellent nutrients for the body (e.g., egg yolk itself), ${ }^{19}$ while others do not.

Other pertinent questions include to what extent are there benefits if indeed increasing dietary cholesterol intake increases total testosterone, which populations would benefit, precisely what dosage would be safe and effective and, lastly, whether a specific food source and period of treatment need to be determined.

Interventions are moreover required that include analysis of serum pregnenolone and (dehydroepiandrosterone) DHEA to ascertain the exact impact of KD dietary cholesterol on the testosterone production pathway.

The next step would be to analyze the KD, or else set up a diet plan incorporating a similar amount of cholesterol to that of the KD, for subjects suffering from male hypogonadism, as well to as to analyze testosterone production accompanying this diet in women, along with resultant libido levels in both genders. 


\section{REFERENCES}

1. Wilson JM, Lowery RP, Roberts MD, et al, 2017 The Effects of Ketogenic Dieting on Body Composition, Strength, Power, and Hormonal Profiles in Resistance Training Males. J Strength Cond Re [Epub Ahead of Print].

2. Lubik AA, Gunter JH, Hendy SC, et al, 2011 Insulin increases de novo steroidogenesis in prostate cancer cells. Cancer Res 71: 5754-5464.

3. Sacks FM, Lichtenstein AH, Wu JHY, et al, 2017 Dietary Fats and Cardiovascular Disease: A Presidential Advisory From the American Heart Association. Circulation 136: e1-e23.

4. Urbain P, Strom L, Morawski L, Wehrle A, Deibert P, Bertz H, 2014 Impact of a 6-week non-energy-restricted ketogenic diet on physical fitness, body composition and biochemical parameters in healthy adults. Nutr Metab (Lond)14: 17.

5. Volek JS, Freidenreich DJ, Saenz C, et al, 2016 Metabolic characteristics of keto-adapted ultra-endurance runners. Metabolism 65: 100-110.

6. Sánchez-Muniz FJ, 2012 Dietary fibre and cardiovascular health. Nutr Hosp 27: 31-45.

7. Key TJ, Roe L, Thorogood M, Moore JW, Clark GM, Wang DY, 1990 Testosterone, sex hormone-binding globulin, calculated free testosterone, and oestradiol in male vegans and omnivores. Br J Nutr 64: 111-119.

8. Traish AM, Testosterone and weight loss: the evidence, 2014. Curr Opin Endocrinol Diabetes Obes 21: 313322.

9. Paoli A, Moro T, Bosco G, et al, 2015 Effects of n-3 polyunsaturated fatty acids $(\omega-3)$ supplementation on some cardiovascular risk factors with a ketogenic Mediterranean diet. Mar Drugs 13: 996-1009.

10. Pérez-Guisado J, Muñoz-Serrano A, Alonso-Moraga A, 2008 Spanish Ketogenic Mediterranean Diet: a healthy cardiovascular diet for weight loss. Nutr J 7: 30.

11. Yancy WS, Olsen MK, Guyton JR, Bakst RP, Westman EC, 2004 A low-carbohydrate, ketogenic diet versus a low-fat diet to treat obesity and hyperlipidemia: a randomized, controlled trial. Ann Intern Med 140: 769-777.

12. Paoli A, Grimaldi K, D’Agostino D, et al, 2012 Ketogenic diet does not affect strength performance in elite artistic gymnasts. J Int Soc Sports Nutr 9: 34.

13. Sawyer JC, Wood RJ, Davidson PW, et al, 2013 Effects of a short-term carbohydrate-restricted diet on strength and power performance. J Strength Cond Res 27: 2255-2262.

14. Bandegan A, Courtney-Martin G, Rafii M, Pencharz PB, Lemon PW, 2017 Indicator Amino Acid-Derived Estimate of Dietary Protein Requirement for Male
Bodybuilders on a Nontraining Day Is Several-Fold Greater than the Current Recommended Dietary Allowance. J Nutr 147: 850-857.

15. Morton RW, McGlory C, Phillips SM, 2015 Nutritional interventions to augment resistance training-induced skeletal muscle hypertrophy. Front Physiol 6: 245.

16. Paoli A, Rubini A, Volek JS, Grimaldi KA, 2013 Beyond weight loss: a review of the therapeutic uses of very-low-carbohydrate (ketogenic) diets. Eur J Clin Nutr 67: 789-796.

17. Knopp RH, Retzlaff BM, Walden CE, et al, 1997 A double-blind, randomized, controlled trial of the effects of two eggs per day in moderately hypercholesterolemic and combined hyperlipidemic subjects taught the NCEP step I diet. J Am Coll Nutr 16: 551-561.

18. McNamara DJ, 2000 The impact of egg limitations on coronary heart disease risk: do the numbers add up? J Am Coll Nutr 19: 5 Suppl: 540-548.

19. DiMarco DM, Norris GH, Millar CL, Blesso CN, Fernandez ML, 2017 Intake of up to 3 Eggs per Day Is Associated with Changes in HDL Function and Increased Plasma Antioxidants in Healthy, Young Adults. J Nutr 147: 323-329.

20. Guasch-Ferré M, Hu FB, Martínez-González MA, et al, 2014 Olive oil intake and risk of cardiovascular disease and mortality in the PREDIMED Study. BMC Med 12: 78.

21. Hunter PM, Hegele RA, 2017 Functional foods and dietary supplements for the management of dyslipidaemia. Nat Rev Endocrinol 13: 278-288.

22. Newman JC, Verdin E, 2014 Ketone bodies as signaling metabolites. Trends Endocrinol Metab TEM 25: 42-52.

23. Schooling CM, Au Yeung SL, Freeman G, Cowling BJ, 2013 The effect of statins on testosterone in men and women, a systematic review and meta-analysis of randomized controlled trials. BMC Med 11: 57.

24. Stanworth RD, Kapoor D, Channer KS, Jones TH, 2009 Statin Therapy Is Associated With Lower Total but Not Bioavailable or Free Testosterone in Men With Type 2 Diabetes. Diabetes Care 32: 541-546.

25. de Keyser CE, de Lima FV, de Jong FH, et al, 2015 Use of statins is associated with lower serum total and non-sex hormone-binding globulin-bound testosterone levels in male participants of the Rotterdam Study. Eur J Endocrinol 173: 155-165.

26. Vidali S, Aminzadeh S, Lambert B, et al, 2015 Mitochondria: The ketogenic diet--A metabolism-based therapy. Int J Biochem Cell Biol 63: 55-59.

27. Rovira-Llopis S, Bañuls C, de Marañon AM, et al, 2017 Low testosterone levels are related to oxidative stress, mitochondrial dysfunction and altered subclinical atherosclerotic markers in type 2 diabetic male patients. Free Radic Biol Med 108: 155-162. 